VeritasE Scientia

Vol. 8. N 1.

Enero - Junio del 2019.

ISSN Edición Online: 2617-0639

https://doi.org/10.47796/ves.v8i1.110

\title{
AFECTACIÓN DEL DERECHO CONSTITUCIONAL A LA INTEGRIDAD PERSONAL DE LAS MUJERES Y LOS INTEGRANTES DEL GRUPO FAMILIAR EN EL PROCESO DE VIOLENCIA FAMILIAR, JUZGADO MIXTO DE ALTO DE LA ALIANZA, TACNA - AÑO 2016.
}

\author{
AFFECTION OF THE CONSTITUTIONAL RIGHT TO THE PERSONAL INTEGRITY OF THE WOMEN AND \\ THE MEMBERS OF THE FAMILY GROUP IN THE FAMILY VIOLENCE PROCESS, MIXED COURT OF ALTO \\ DE LA ALIANZA, TACNA-YEAR 2016.
}

Charles Harrinson Quiñones Talledo ${ }^{1}$

Carmen Ruth Álvarez Goicochea²

Presentado: $18 / 04 / 2019$

Aceptado: $17 / 06 / 2019$

Publicado online:17/07/2019

\section{RESUMEN}

Se llevó a cabo una investigación respecto de la afectación del derecho constitucional de la integridad personal de las mujeres y los integrantes del grupo familiar en el proceso de violencia familiar en el juzgado mixto de Alto de la Alianza de Tacna en el año 2016, en consideración de la ley N³0364. Se analizó 218 expedientes de violencia familiar y se aplicaron 127 encuestas a las víctimas que se veían afectadas en su integridad personal. La investigación pretende demostrar la existencia de afectación del derecho constitucional a la integridad personal y respecto de la operatividad de las medidas de protección para la salvaguarda de la integridad personal de las víctimas y entorno a su eficacia, pues para aproximadamente la mitad de las víctimas, no evitaron la reincidencia o nuevas agresiones por parte de los agresores. Sin embargo, se hace necesario implementar una propuesta técnica que contengan nuevos procedimientos jurídicos y administrativos que permitan la determinación un mejor procedimiento tanto en el seguimiento y control de su aplicación para la protección de las víctimas. Dicha propuesta contempla el otorgamiento de una mayor participación del Juzgado Mixto o Módulo de Familia de la Corte Superior de Justicia de Tacna (CSJT). Asimismo, la propuesta elaborada considera la creación del puesto de Abogado Protector, quien estaría adscrito al Módulo de Familia como parte del equipo multidisciplinario y se encargaría de verificar el cumplimiento de las medidas de protección y la salvaguarda de la integridad personal como derecho fundamental de las víctimas. La reforma de la aplicación de las medidas de protección contempla nuevos mecanismos

${ }^{1}$ Magíster en Derecho Constitucional

2 Doctora en Derecho, Académica de la Escuela de Postgrado de la Universidad Privada de Tacna 
jurídicos y administrativos que deberían ser recogidos en una ley de modificatoria del reglamento de la violencia contra las mujeres y los integrantes del grupo familiar actualmente vigente, dado que existe en gran medida afectación a la integridad personal de las víctimas.

Palabras clave: violencia, juzgado de familia, violencia psicológica, víctimas e integridad.

\begin{abstract}
An investigation was carried out regarding the affectation of the constitutional right of the personal integrity of the women and the members of the family group in the process of family violence in the joint high court of the Tacna alliance in 2016, in consideration of Law No. 30364. 218 family violence cases were analyzed and 127 surveys were applied to the victims who were affected in their personal integrity. The investigation aims to demonstrate the existence of the violation of the constitutional right to personal integrity and regarding the operation of protective measures to safeguard the personal integrity of the victims and their effectiveness, as for approximately half of the victims, they did not avoid recidivism or new aggressions on the part of the aggressors. However, it is necessary to implement a technical proposal that contains new legal and administrative procedures that allow the determination of a better procedure both in the monitoring and control of its application for the protection of victims. Said proposal contemplates the granting of a greater participation of the Mixed Court or Family Module of the CSJT. Likewise, the elaborated proposal considers the creation of the position of Protective Lawyer, who would be attached to the Family Module as part of the multidisciplinary team and would be responsible for verifying compliance with the protection measures and the safeguarding of personal integrity as a fundamental right of the victims. The reform of the application of the Protection Measures contemplates new legal and administrative mechanisms that should be included in a law amending the regulation of violence against women and members of the family group currently in force, given that there is a great deal of impact on the personal integrity of the victims.
\end{abstract}

Keywords: violence, family court, psychological violence, victims and integrity.

\title{
INTRODUCCIÓN
}

La afectación del derecho constitucional a la integridad personal se desplaza a través del tiempo como una constante causando graves afectaciones tanto físico como psicológico y muy particularmente en la región Tacna, violencia que se genera contra las mujeres y los integrantes del grupo familiar, convertido en una situación frecuente. Es necesario destacar que, frente a la afectación del derecho constitucional a la integridad personal de las víctimas en sus diferentes variables, estas se enmarcan en los tipos de afectación física y psicología en un margen amplio, sobre el cual el Estado Peruano no puede optar por una posición inactiva y así permitir que sus efectos sigan dañando el tejido social el país y de la región. Se dio origen a fines del año 2015 a la promulgación de la "Ley para prevenir, sancionar y erradicar la 
violencia contra las mujeres y los integrantes del grupo familiar" (Ley $N^{\circ} 30364$ ), dotada de medidas de protección a favor de las víctimas, para proteger el derecho constitucional a la integridad personal, facultando a los magistrados de familia el otorgar medidas de protección a las víctimas directas e indirectas en donde se ven afectadas en su integridad personal, a fin de frenar la frecuencia de los tipos de agresiones físicas y psicológicas contra las mujeres y de los integrantes del grupo familiar, siendo el Ministerio Público y, especialmente, la Policía Nacional, los entes encargados de hacer cumplir las disposiciones emanadas por los juzgados de familia para proteger a las víctimas en cuanto a la medida de protección, de cualquier tipo de violencia: ya sea sexual, económica, psicológica o física. Las medidas de protección son otorgadas por los jueces mixtos y de familia, sin embargo, carecen ellas de eficacia en su procedimiento, lo que incide en la reincidencia de aquellas expresiones lesivas al bienestar, no solo sobre la dignidad como ser humano sino en las afectaciones del derecho constitucional a la integridad personal, que significa un menoscabo en su salud física y mental. En el devenir del tiempo, la afectación del derecho constitucional a la integridad personal, se presenta como uno de los problemas sociales más antiguos de la humanidad, se ha convertido a través del tiempo en una constante en la actualidad, cuya erradicación parece postergarse en el tiempo, siendo ahora, el tema que ocupa un lugar no menos interesante o importante, en el que hacer de un Estado Constitucional de Derecho.

La nueva ley de violencia familiar no 30364, ley para prevenir, sancionar y erradicar la violencia contra las mujeres y los integrantes del grupo familiar, tampoco ha resuelto el problema de la frecuencia de violencia que va en aumento, en donde se evidencian la existencia de la afectación del derecho constitucional a la integridad personal de las mujeres y de los integrantes del grupo familiar. Si partimos de la premisa que la protección del derecho constitucional a la integridad personal se da mucho antes de que se genere una identidad definida, como en el caso del concebido es sujeto de derecho en todo cuanto le favorece. Es lamentable que en el inicio de un nuevo siglo la Dogmática del Derecho Constitucional, siga discutiendo sobre si el matrimonio es o no contrato o sobre si la familia es una institución solamente jurídica o social, que al parecer por mucho tiempo estos conceptos han sido dejados atrás en la comunidad internacional. En nuestra realidad local se observa con cierto desaliento que las medidas de protección que son generados por tipos de violencia en clara afectación del derecho constitucional a la integridad personal de las mujeres y los integrantes del grupo familiar, que establece la ley no 30364, están sólo en papel y no se cumplen en la práctica, por cuanto muchas veces, pese a que están dictadas por un órgano Jurisdiccional, y que por tanto deberían cumplirse obligatoriamente, no es realmente así. Muchas veces el agresor se burla de la ley y vuelve a cometer actos de violencia contra la víctima, en otras oportunidades el agresor se esconde y burla la autoridad de la ley, que pocas veces tiene medidas efectivas, carece de seguimiento y control por parte de órganos de auxilio que hagan cumplir de manera correcta y efectiva sus mandatos. Por lo general, el agresor tiende a incumplir los mandatos del Juzgado por cuanto se percata que éstos pueden ser burlados fácilmente, no existe un adecuado tratamiento y uso del mapa georeferencial-situacional de la víctima, ello, porque no existen medidas coercitivas que les exija e impongan el cumplimiento obligatorio de sus mandatos.

Existen muchos casos de afectación del derecho constitucional a la integridad personal de las mujeres y los integrantes del grupo familiar en los que se han puesto en práctica las medidas de protección establecidas; sin embargo, como se ha manifestado, existe burla en su cumplimiento por parte de los agresores, quienes generalmente son personas que carecen de valores, de escasa educación, respeto del cual el Estado no tiene operatividad alguna, es decir, no existe un tratamiento adecuado por parte de las instituciones públicas que sean capaces de generar un soporte moral de valores, educación, lo cual debe de fortalecerse y sentar bases desde la formación educativa inicial hasta finalizar en la superior y orientar a la población a la iniciativa de un auto aprendizaje constante, sobre todo de los niños que al fin del cabo serán los futuros adultos, y si no tienen soporte moral, ético y espiritual, los actos de violencia continuarían siendo parte de las estadísticas que van en aumento sin freno, sin medida, lo cual hace que el agresor quede impune e inclusive se torne más violento, inconsciente del mal que genera y del daño 
que causa al entorno familiar, poniendo a la víctima en situación de vulnerabilidad e inferioridad. Magaña de la Mora (2017) refiere que España y México han debido adecuar su normatividad y práctica judicial a obligaciones internacionales que protegen la familia, así como a sus integrantes, del fenómeno de la violencia familiar. Morales y Sandrini (2010) concluyen que la situación de violencia intrafamiliar sólo es considerada para determinar algunos aspectos del delito, lo que perjudica la posición de las mujeres víctimas de violencia frente al proceso penal y la aplicación de la normativa vigente.

Laguna (2015) propone que nuevos órganos mantengan competencia por razón de las personas únicamente cuando se produzcan actos de violencia que afectan la integridad personal de las víctimas, entre marido y mujer o parejas con análoga relación de afectividad, tanto heterosexual como homosexual. Orna-Sánchez (2013) enfatiza la trascendencia de la violencia familiar a nivel internacional y nacional y su repercusión social tanto en el curso de la existencia como en la realización del ser humano en el que se ven afectadas en su integridad personal las víctimas. Cervantes (2010) enfatiza la trascendencia de la violencia psicológica, el daño al proyecto de vida, y su repercusión en el curso de la existencia y en la realización del ser humano. Se ha considerado que la violencia familiar es una forma de relación disfuncional en la familia que causa daño a la persona en su integridad como víctima. El Trastorno de Estrés Post Traumático es el cuadro clínico que mejor describe a las víctimas de violencia familiar, se enfatizan los síntomas de reexperimentación e hiperactivación. Calisaya (2017) concluye que no son idóneas las medidas de protección en el que se ven afectas en su integridad personal las víctimas, dictadas por el Juez de Familia debido a que la Policía Nacional del Perú remite atestados policiales que no contienen un buen material informativo, existe una deficiente participación de la propia víctima en la investigación y la vigencia de la medida de protección se encuentra condicionada a lo que resuelva el Juez Penal o el Juez de Paz letrado. Pizarro-Madrid (2017) afirma que las medidas de protección reguladas en la Ley N 30364 "Ley para Prevenir, Sancionar y Erradicar la Violencia Contra las Mujeres y los Integrantes del Grupo Familiar" no tienen naturaleza jurídica cautelar, anticipada, genérica y autosatisfactiva, tan sólo posee algunas características propias de las mismas.

Gonzáles (2011) afirma que la violencia familiar es una violación de los derechos humanos porque afecta la integridad personal, la salud de la víctima, incluida su integridad emocional, ya que el ser humano es un todo, y su bienestar le permite ser más productivo y sus relaciones con su entorno, entre ellos su familia, de manera sana, hoy en día la violencia familiar, ha sido considerada como un problema de salud pública. Fuentes, (2016) refiere que la mayoría de los profesionales en familia han manifestado que los factores jurídicos que se asocian al incremento de violencia familiar es la ineficacia de las medidas de protección entorno a la afectación de la integridad personal como víctimas; ya que presentan debilidades que faltan regular. Rodríguez (2013) encontró que existe relación de incidencia significativa entre el tratamiento de las medidas de protección, dictadas por el Ministerio Público y la Violencia Familiar entre Cónyuges y Convivientes, en el Distrito Judicial de Tacna. Yáñez (2015) afirma que la violencia familiar, constituye una violación de derechos humanos y libertades fundamentales de las personas, en tal sentido limita total o parcialmente a la víctima en el disfrute y ejercicio legítimo de los derechos a la dignidad, integridad y libertad personal previstos en los artículos 1, 2.1 y 2.24. de la Constitución Política del Estado.

\section{OBJETIVOS}

Identificar el tipo de afectación del derecho constitucional de la integridad personal de las mujeres y los integrantes del grupo familiar en proceso de violencia familiar del Juzgado Mixto de Alto de la Alianza de Tacna en el año 2016.

Proponer procedimientos técnicos que permitan un mejor seguimiento y control de las víctimas afectadas en su derecho constitucional a la integridad personal a fin de lograr la eficacia de medidas de protección. 


\section{METODOLOGÍA}

Investigación no experimenta, observacional analítica que usa el método deductivo. Permite hacer una comparación entre las diversas legislaciones existentes relacionadas al tema, sobre la afectación del derecho constitucional a la integridad personal, para de esta manera enriquecer nuestra legislación interna. Utiliza el método hermenéutico para interpretar las normas y principios, buscando su verdadero sentido a fin de ser utilizado de manera coherente, congruente y sistemática. La investigación se llevó a cabo en el Juzgado Mixto de Alto de la Alianza (MBJAA) perteneciente a la Corte Superior de Justicia de Tacna. La técnica documental se aplicó a los expedientes en los procesos de violencia familiar contra las mujeres y los integrantes del grupo familiar tramitado en el Juzgado Mixto de Alto de la Alianza. La técnica de la encuesta (dirigidas a las víctimas) también se aplicó en este juzgado. El tiempo social de investigación se realizó a partir del mes de enero a diciembre del año 2016. Se seleccionó una muestra aleatoria de 218 expedientes

\section{RESULTADOS}

En relación a la edad de la víctima en las que se ven afectadas en su derecho constitucional a la integridad personal, resulta que las de mayor incidencia es la de 31 a 40 años (37.8\%); seguida las de 24 a 30 años (29.1\%); en tercer lugar, entre 41 a 50 años con un porcentaje (16.5\%) (Fig.01)

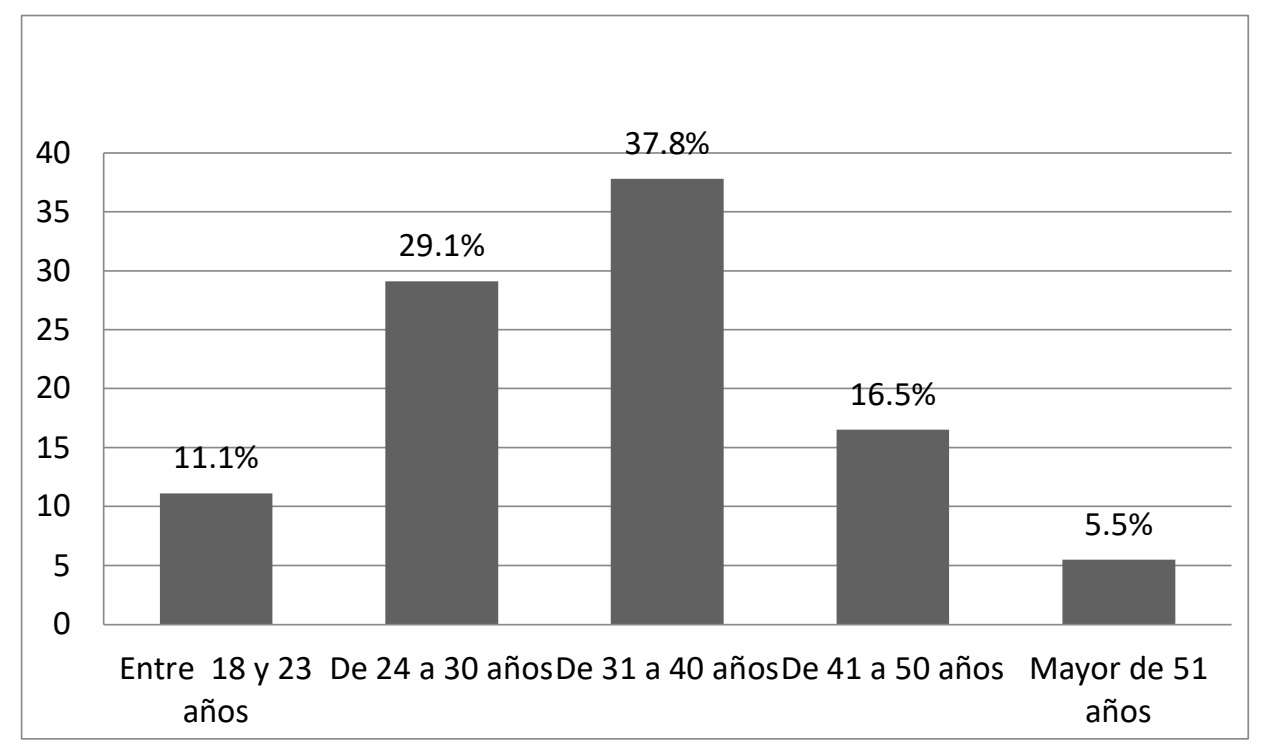

Figura 1: Edad de las víctimas de violencia familiar

En cuanto a la condición del estado civil se tiene los grupos de mayor incidencia en número de 73 son convivientes (57.5\%) seguidas por 20 víctimas que tienen la condición de separadas o divorciadas (15.7\%). En el caso de denuncias por primera vez, fueron 111 las agraviadas (87.4\%). En cuanto a las veces que se presentaron denuncias el mayor número refiere que hasta 3 veces (54.9\%); seguidas de las que denunciaron hasta en 2 oportunidades (25.2\%).

En cuanto al tipo de violencia la psicológica, el 40.9\% de las afectadas tuvo este antecedente; seguido una afectación de tipo económica en el $27.6 \%$, en tercer las víctimas que han sufrido una afectación de tipo física en el $22.8 \%$ y finalmente la afectación de tipo sexual en un $8.7 \%$.

En cuanto a la incidencia de lo dispuesto por la autoridad judicial relacionado al alejamiento de las personas agresoras del entorno de la víctima y de su hogar, 125 casos tuvieron esta situación final.

El 55,2\% la persona agresora no cumplió con la orden judicial dispuesta en la medida de protección ante la existencia de afectación del derecho constitucional a la integridad personal de las víctimas, es decir, de alejarse el agresor de las agraviadas 
En cuanto si en la actualidad la victima reside con la persona agresora, un número de 86 víctimas refirió que no (67.7\%); siendo en menor margen aquellas víctimas en que refirieron que si residen con la persona agresora (32.3\%). En cuanto si la persona agresora es una amenaza para la integridad de la víctima, nos refieren que, sí constituye una amenaza la persona agresora, (95.3\%).

Existe la afectación del derecho constitucional a la integridad personal de las víctimas de las mujeres y los integrantes del grupo familiar, asociadas estas con las medidas de protección dictadas: Se tiene que el tipo de afectación en la integridad personal fue primeramente de tipo psicológico, seguidas del tipo de afectaciones de tipo físicas, generalmente su agresor viene a ser su conviviente, seguido en número de reducido de esposos, ex parejas, tío, sobrino o hijos, dictándose como medida de protección el prohibir de ejercer cualquier tipo de violencia a las víctimas, llegando a tenerlas las víctimas hasta 04 días de incapacidad médico legal como afectación en su integridad personal.

Desde la aplicación de la ley 30364, de fecha 23 de noviembre del 2015, en cuanto al aporte de los gobiernos regionales o locales, no están operativas puesto que en el caso de las DEMUNAS, que comprende la actuación de las Municipalidades los casos de violencia familiar una vez toman conocimiento, estos son derivados al CEM-Centro de Emergencia Mujer (ente del Ministerio de la Mujer y Poblaciones Vulnerables), donde la parte agraviada presente afectación psicológica ponen de conocimiento al juzgado de familia para la medidas de protección y en el caso de existir agresiones físicas son puestos de conocimiento a las dependencias de policía nacional (comisaria), a efectos de que la parte agraviada pase a revisión médico legal a efectos de determinar los días de incapacidad médico legal, se determine el tipo de afección sufrida, que una vez recabado ello, son puestos de conocimiento al juzgado de familia a fin de que se emita la correspondiente medida de protección. De otra parte, en cuanto al registro único de víctimas y agresores, la norma precisa en el artículo 42 del texto legal hace mención a un sistema intersectorial de registro de casos de violencia contra las mujeres y los integrantes del grupo familiar, el ministerio público en coordinación con la Policía Nacional del Perú, el Poder Judicial y el Ministerio de la Mujer y Poblaciones Vulnerables, son responsables del registro de los caso, sin embargo, en el caso de la Comisaria de Alto Alianza, no se lleva ni se cuenta con un registro a fin de efectivizar el procedimiento que comprenda las causas, denuncias anteriores y demás datos necesarios, no se cumple, puesto que tampoco se realiza el seguimiento y control adecuado con un mapa georeferencial situacional de la víctima o sobre todo garantizar que el resultado sea optimo, en cuanto la aplicación adecuada de las medidas de protección en cuanto a su estricto cumplimiento, según los dispuesto por el juzgado de familia.

El 44.8\% de las víctimas señalaron que los agresores sí cumplieron con respetar las medidas de protección dictadas a favor de las víctimas. Mientras que el 55.2\% respondió que los agresores no cumplieron con dicha medida de protección. Asimismo, se halló que el 63.2\% de las víctimas señalaron que los agresores no volvieron a agredirlas por lo que se evidencia su cumplimiento de las medidas de protección para este $63.2 \%$ de las víctimas. Mientras que para el $36.8 \%$ no se logró un resultado óptimo durante la ejecución de las medidas de protección dictadas a su favor, lo que resulta que continúa existiendo la afectación del derecho constitucional a la integridad personal de las mujeres y los integrantes del grupo familiar, hecho reiterado, siendo importante el reflejo de ello. La persona agresora aumentó la frecuencia de violencia familiar contra las víctimas después que la autoridad judicial dicto medida de protección a su favor en un número de 62 (48.8\%).

Procedimientos técnicos ante la existencia de afectación del derecho constitucional a la integridad personal de las mujeres y los integrantes del grupo familiar en proceso de violencia familiar.

En la determinación de los procedimientos técnicos que se toman una vez producida la afectación del derecho constitucional a la integridad personal de las víctimas, asociados estos a través de las medidas de protección dictadas a favor de las víctimas, se tienen los siguientes: 
Antes de dictar las medidas de protección se evidencia la existencia de afectación del derecho constitucional a la integridad personal de las víctimas, ante lo cual se debe evaluar la peligrosidad de los agresores (la actual ley y su reglamento no contempla este aspecto)

La policía nacional debe realizar entrevistas periódicas a las víctimas en que existe afectación del derecho constitucional a la integridad personal y no esperar que se vuelvan a presentar denuncias, pues en muchas ocasiones, las víctimas viven en un ambiente de amenazas por parte de sus agresores y por ello, no se atreven a volver a denunciar a sus agresores, siendo que en algunos casos las víctimas no se presentan para ser examinadas por lo que las denuncias concluyen en no ha lugar el dictar medida de protección, situación que las coloca en un estado de vulnerabilidad y riesgo.

Debe involucrarse a las organizaciones comunitarias o vecinales en el seguimiento, control y verificación del cumplimiento de las medidas de protección ante la existencia de afectación de la integridad personal como derecho fundamental de las víctimas.

Asimismo, los propios juzgados de familia y juzgados mixtos pueden citar en forma aleatoria a las agraviadas ante la existencia de afectación del derecho constitucional a la integridad personal de las víctimas, para realizar breves entrevistas y así verificar el cumplimiento de las medidas de protección dictadas a su favor.

En las agraviadas en que exista afectación del derecho constitucional a la integridad personal, deben ser instruidas para aportar pruebas materiales y no solo sus testimonios al momento de presentar nuevamente sus denuncias por agresiones de cualquier tipo por parte de su agresor.

La no asistencia del denunciado a las pericias psicológicas debe considerarse como un factor de riesgo en contra de las víctimas, al igual que no presentación de las víctimas para ser examinadas y ello, debe tener presente al momento de dictar las medidas de protección.

Finalmente, ante la existencia de afectación del derecho constitucional a la integridad personal de las mujeres y los integrantes del grupo familiar, en gran medida está asociada al resultado de las medidas de protección dictadas a favor de las víctimas, ante lo cual se hace necesario el verificarse en el lugar mismo en que se suscita, es decir, en los propios hogares con un mapa georeferencial y situacional de las víctimas. Para ello, además del uso de llamadas telefónicas en un periodo semanal a las víctimas que se ven afectadas en su integridad personal para recoger sus testimonios sobre el cumplimiento de las medidas de protección, debería crearse un equipo multidisciplinario que solo tenga por objetivo hacer seguimiento y control de los casos de violencia familiar. Este equipo hará la verificación precisa en cuanto al resultado de las medidas de protección dictadas.

Criterios para reformar la aplicación de las medidas de protección para delimitar sus alcances funcionales ante la existencia de afectaciones del derecho constitucional a la integridad personal.

Se plantea la necesidad de formular los criterios objetivos para reformar o reformular la aplicación de las medidas de protección en los que exista afectación del derecho constitucional a la integridad personal de las víctimas para delimitar sus alcances funcionales. Antes de formular nuevos mecanismos para la aplicación de un plan efectivo las medidas de protección, se considera pertinente señalar algunos propios en los cuales deben basarse:

Principio de debida diligencia

Principio de protección a la víctima de violencia y víctimas indirectas

Principio de no discriminación

Principio de urgencia o inmediatez

Principio de simplicidad

Condiciones previas para el otorgamiento de medidas de protección ante la existencia de la afectación del derecho constitucional a la integridad personal de las mujeres y los integrantes del grupo familiar en proceso de violencia familiar. 
1.- Para todos los casos de solicitud de Medidas de Protección, se nombrará a un Abogado de Protección (adscrita al Módulo Mixto o de Familia de la CSJT) que tramitará y dará seguimiento al cumplimiento y control de las Medidas. Dicho abogado se encontrará adscrito al Equipo Multidisciplinario del Módulo de Familia de la CSJT.

2.- Todos los trámites de las Medidas de Protección serán realizados por el Equipo Multidisciplinario, adscrito a la CSJT, ejecutando las acciones encaminadas para valorar el riesgo de daño en la víctima, recabar las pruebas y hacer tramitación, definiendo el tipo de orden y su objetivo, que se solicitará ante la CSIT. Al ser de tipo de emergencia y/o preventivas, se procurará realizar todas las diligencias para su cumplimiento en un máximo de 24 horas hábiles.

3.- Se prestará medidas especiales para garantizar el acceso a las Medidas de Protección en condiciones de igualdad y no discriminación, cuando la usuaria además de ser víctima de violencia sea: niña, indígena, tenga alguna discapacidad, migrantes o tenga otra condición que indique un factor de mayor riesgo y vulnerabilidad.

4.- Las medidas alternativas a las Resoluciones Judiciales de Protección y/o el plan de seguridad se facilitarán a la víctima en todo momento, con independencia de que se tramiten o concedan las Medidas de Protección.

5.- En el trámite de la Resolución, se dará prioridad aquellas que causen una menor molestia a la víctima y víctimas indirectas. Es decir, que la Resolución de Medidas de Protección se encauce a garantizar la integridad personal de la víctima, permanezca en el domicilio, asegurando la desocupación por parte del agresor.

6.- El Abogado de Protección solicitará las Medidas de Protección de naturaleza civil/familiar, además de su seguimiento en la vía penal, complementarias y coadyuvantes a las de emergencia/preventivas para garantizar el derecho constitucional a la integridad personal de las víctimas y la máxima seguridad de la víctima.

Cumplimiento de las Medidas de Protección ante la existencia de afectación del derecho constitucional a la integridad personal de las víctimas.

Llevar a cabo, desde que se concede la Resolución de las Medidas de Protección ante la existencia de afectación del derecho constitucional a la integridad personal de las víctimas, todas las acciones necesarias para verificar y coadyuvar en el cabal cumplimiento de la resolución judicial, garantizando que en caso de incumplimiento se priorice la protección y seguridad de la víctima y víctimas indirectas entorno a la integridad personal en consideración que es un derecho constitucional fundamental de las víctimas y de vital importancia; así como la sanción a las autoridades o particulares que no dieron cumplimiento a la Resolución Judicial.

Secuencia de la Aplicación de la Resolución de las Medidas de Protección ante la existencia de afectación del derecho constitucional a la integridad personal de las víctimas.

1. La Resolución de Protección ante la existencia de afectación del derecho constitucional a la integridad personal de las víctimas, concedida por el o la juez/a tiene una duración de 72 horas.

2. El Abogado de Protección encargado del caso realizará acciones para verificar y coadyuvar en el cumplimiento de la Orden de Protección existiendo afectación del derecho constitucional a la integridad personal de las víctimas.

3. Para el cumplimiento de las Medidas de Protección y dependiendo del tipo de Resolución concedida existiendo afectación del derecho constitucional a la integridad personal de las víctimas, el Módulo de Mixto o el de Familia contará con vehículos de transporte adecuados que faciliten el traslado de la víctima y las víctimas indirectas; así como la recuperación de sus objetos y bienes.

4. Se gestionará desde el trámite de solicitud de la orden que la víctima se mantenga en su domicilio garantizando el desalojo del agresor. 
5. Se procurará que la o el juez se encuentre presente en las diligencias para el cumplimiento de la Resolución de las Medidas de Protección ante la existencia de afectación del derecho constitucional a la integridad personal de las víctimas.

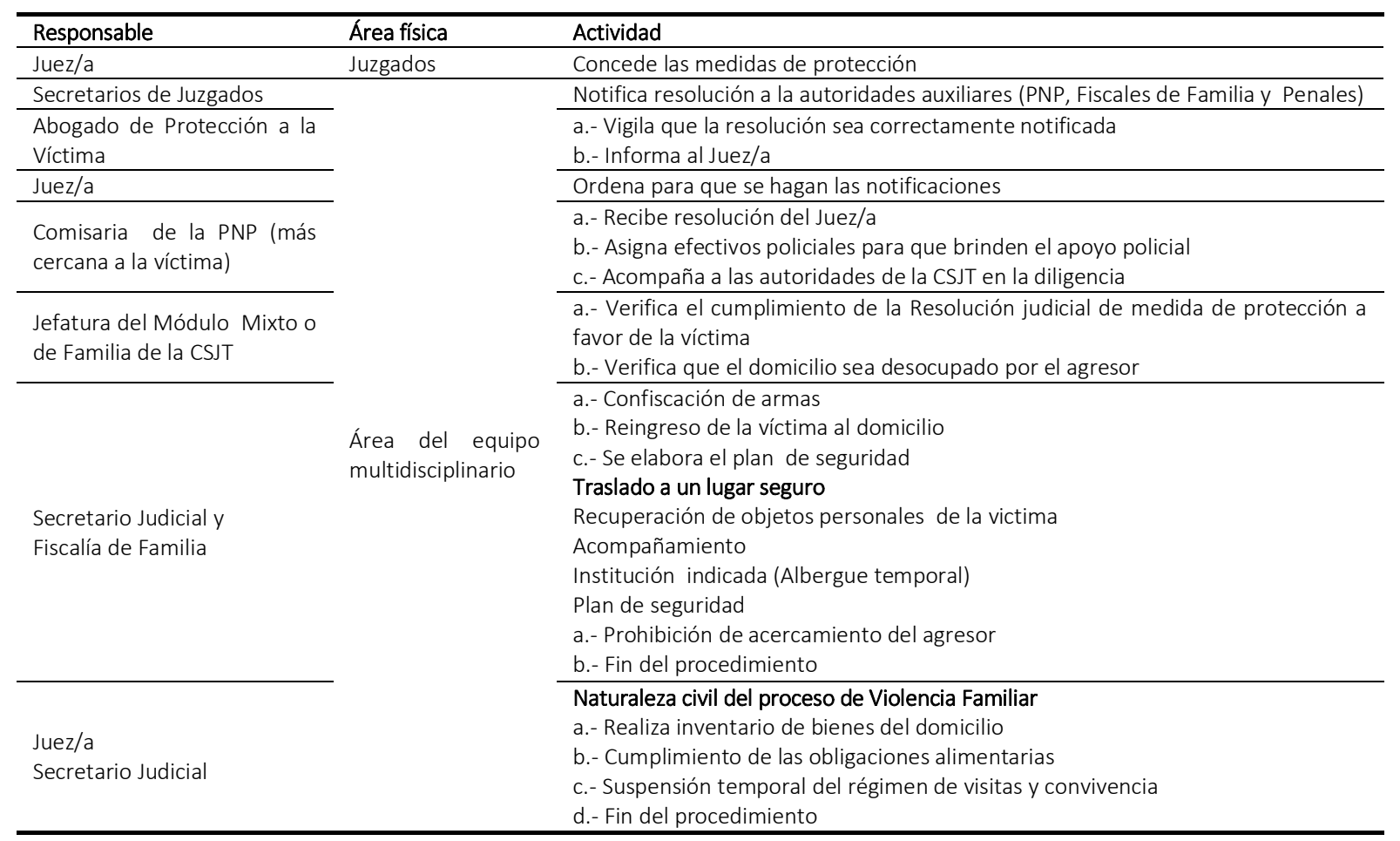

Figura 1 Actividades en el otorgamiento de las Medidas de Protección.

Control y seguimiento a las Medidas de Protección ante la existencia de afectación del derecho constitucional a la integridad personal de las víctimas.

Supervisar el adecuado cumplimiento de las Medidas de Protección ante la existencia de afectación del derecho constitucional a la integridad personal de las víctimas por medio de un sistema de control y seguimiento, a través del monitoreo del Módulo Mixto o de Familia y del seguimiento que realice el Abogado de Protección. Asimismo, realizar una valoración del riesgo al finalizar el cumplimiento de las Medidas de Protección a fin de determinar la pertinencia de continuar con la protección o cerrar temporalmente el expediente.

\section{Actividades}

1. El Abogado de Protección establecerá comunicación con la víctima cada 24 horas para verificar su seguridad y el estado ante la existencia de afectación del derecho constitucional a la integridad personal de las víctimas y que tenga como resultado el cumplimiento de la Orden de Protección.

2. Mediante el mapa geo referencial de los lugares que habita o frecuenta la víctima, se mandará un aviso a la Comisaría del Sector más cercano a que brinde vigilancia continua mediante patrullajes policiales que se realizarán por lo menos cada ocho horas.

3. Cuando esté por vencerse el plazo de duración de las Medidas de Protección, con un mínimo de 12 horas de anticipación, se tendrá que volver a valorar el riesgo para determinar la situación de peligro en que se encuentra la víctima. 
4. Al margen de la acción que se determine al término de la Resolución de las Medidas de Protección, se mantendrá el Plan de Seguridad hasta mínimo un mes, pudiéndose prolongar el tiempo que necesite la víctima para recuperar la confianza y la seguridad.

\section{DISCUSIÓN}

Más de un tercio de las víctimas de violencia familiar señalan que han continuado las agresiones, sobre todo de tipo psicológico, seguidas las de tipo físicas, aún después de verse afectadas en su integridad personal y de otorgadas las medidas de protección. A pesar del alejamiento del agresor y de la prohibición de que el agresor se acerque a la víctima, muchas mujeres continúan siendo víctimas puesto que refieren haber sido nuevamente objeto de agresiones de tipo psicológica por parte de sus cónyuges o agresores. Por lo tanto, la existencia de afectación del derecho constitucional a la integridad personal de las mujeres y los integrantes del grupo familiar es altamente notable y la situación de agresión que se repite de manera continua. En lo que comprende el Juzgado Mixto de Alto de la Alianza de Tacna durante el año 2016, se analizaron y procesaron como parte de la presente investigación un numero d 218 expedientes tramitados por proceso de violencia familiar, resultando en gran medida que si existe la afectación del derecho constitucional a la integridad personal de las mujeres y los integrantes del grupo familiar, siendo mayormente agresiones de tipo psicológicas, seguido de afectaciones de tipo físico, en el que las víctimas son en su mayor número mujeres las que se ven afectadas en su derecho constitucional a la integridad personal, en un escaso margen varones y menores de edad como afectados; lo cual también es corroborado con las encuestas a las víctimas en las que mayormente sufren afectaciones de tipo psicológica. Se ha elaborado una propuesta técnica que contempla como nuevo procedimiento jurídico y administrativo que permitan la determinación de un mejor resultado entorno a las medidas de protección ante la existencia de afectación del derecho constitucional a la integridad personal de las mujeres y los integrantes del grupo familiar en proceso de violencia familiar. Dicha propuesta contempla el otorgamiento de una mayor participación del Juzgado Mixto y el Módulo de Familia, con participación de los integrantes del Equipo Multidisciplinario de la CSJT. Asimismo, la propuesta elaborada considera la creación del puesto de Abogado Protector, quien estaría adscrito al Juzgado Mixto o el Módulo de Familia y se encargaría de verificar y supervisar el cumplimiento de las medidas de protección ante la existencia de afectación del derecho constitucional a la integridad personal de las víctimas.

Es recomendable que se lleven a cabo el diagnóstico del mapa georeferencial de las zonas de alto riesgo y situación de vulnerabilidad, con un trabajo de monitoreo y actualización constante para su prevención y erradicación, en consideración de un Plan de Seguridad hasta mínimo por un mes, pudiéndose prolongar el tiempo que necesite la víctima para recuperar la confianza y la seguridad, puesto que los índices de proceso de violencia familiar van en aumento y se ha convertido en un problema de salud pública, para lo cual se hace necesario también el evaluar la peligrosidad de los agresores, que la actual ley y su reglamento no contempla, a fin de que se identifiquen los vacíos o fallas para la obtención de resultados satisfactorios. El Juzgado Mixto de la Alianza de Tacna debe realizar convenios con otras instituciones públicas del distrito para coadyuvar no solo fortalecer la vigilancia, seguimiento y verificación o control del cumplimiento de las medidas de protección ante la existencia de afectación del derecho constitucional a la integridad personal de las mujeres y los integrantes del grupo familiar en proceso de violencia familiar, sino también debe considerar como meta primordial el enfoque hacia una educación formativa con el objeto de proporcionar conocimiento adecuado en todos sus niveles educativos, sobre la no generación de violencia en sus diferentes variables. Se recomienda que el Módulo de Familia tenga la posibilidad de contratar a un "abogado protector", quien estaría abocado a hacer seguimiento y control de las medidas de protección dictadas y además, de asegurarse el cumplimiento del alejamiento del agresor de la víctima y de la coordinación con otras instituciones y profesionales para hacer efectiva y eficaz las medidas de protección dictadas. 


\section{REFERENCIAS BIBLIOGRÁFICAS}

Alonso, E. (2007). Mujeres víctimas de violencia doméstica con trastornos de estrés postraumático: Validación empírica de un programa de tratamiento. Tesis doctoral. Universidad Autónoma de Madrid. Madrid. España.

Álvarez; J. (2004). Familia, integración, conflicto y violencia. Estudio en el Estado de Tamaulipas. Gobierno del Estado de Tamaulipas. México: Edit. DIF Tamaulipas, Dirección de Comunicación social. México.

Amor, P. Echeburúa, E., Paz de Corral, Sarasua, B. y Zubizarreta, I. (2001). Maltrato físico y maltrato psicológico en mujeres víctimas de violencia en el hogar: un estudio comparativo. Revista de Psicopatología y Psicología Clínica. 2001, Volumen 6. Número 3 pp. 167-178. Asociación Española de Psicología Clínica y Psicopatología (AEPCP).

Arriola, I.S. (2013). Obstáculos en el acceso a la justicia de víctimas de violencia psicológica en el procedimiento de violencia familiar nacional. ¿Decisiones justas con enfoque de derechos humanos y de género? Análisis de casos con resolución de la Segunda Sala de Familia de Lima entre setiembre - diciembre 2011". Tesis para optar el grado de Magíster en Derechos Humanos. Escuela de Posgrado. Pontificia Universidad Católica del Perú.

Asensi, L. (2008). La prueba pericial psicológica en asuntos de violencia de género. Revista Internauta de Práctica Jurídica. Núm. 21, año enero-junio 2008, págs. 15-29, 15. Comunidad Valenciana. España.

Benítez, M. (1999). Violencia doméstica en el municipio de Albacete. Ayuntamiento de la mujer. Consejería de Albacete. España.

Bernales, E. y Otárola, A. (1996), La Constitución de 1993. Análisis comparado, Lima, Ed. Konrad Adenauer, Ciedla, 88.

Blázquez, M., Moreno, J. y García-Baamonde, M.E. (2010). Revisión teórica del maltrato psicológico en la violencia conyugal. Psicología y Salud, Vol. 20, Núm. 1: 65-75, enero-junio de 2010

Cabanellas, G. (2006). Diccionario jurídico elemental. (8 tomos, $25^{\circ}$ Ed.). Buenos Aires: Editorial Heliasta

Calisaya, P. (2017). Análisis de la idoneidad de las medidas de protección dictadas a favor de las víctimas de Violencia en el primer juzgado de familia de Puno, Periodo noviembre de 2015 a noviembre de 2016 en el Marco de la ley 30364 ley para prevenir, sancionar y erradicar la violencia contra las mujeres y los Integrantes del grupo familiar. Tesis de Derecho. Escuela Profesional de Derecho. Universidad Nacional del Altiplano. Puno.

Camargo, P. (1974), La problemática mundial de los derechos humanos. Bogotá: Editorial Retina, 44.

Cervantes, V. (2010). Análisis jurídico descriptivo de la violencia familiar y el daño a la persona en el derecho civil peruano. Revista de la Facultad de Psicología-UNMSM. Recuperado de: http://revistasinvestigacion.unmsm.edu.pe/index.php/psico/article/view/3741/300.

CIDH, Caso Martín de Mejía c. Perú, (1995), No. 10.970, Informe 5/96, de 01 de marzo de 1996, publicado en Informe Anual de la Comisión Interamericana de Derechos Humanos,168-214 (200-201).

Condori, M. y Guerrero, P. (2010). Factores individuales, sociales y culturales que influyen en la violencia basada en género en mujeres de 20 a 64 años de edad en el Centro de Salud Ganímedes San Juan de Lurigancho octubre - diciembre de 2010. Tesis Para optar el título profesional de Licenciada en Obstetricia. Universidad Nacional Mayor de San Marcos. Lima. Recuperado de: http://cybertesis.unmsm.edu.pe/bitstream/cybertesis/2993/1/Condori_fm.pdf

Contreras, S. (2012). Ferrajoli y su teoría de los derechos fundamentales. Estudios de filosofía práctica e historia de las ideas, 14(2), 17-28.

Compilación de Instrumentos Internacionales (2001), Oficina en Colombia del Alto Comisionado de las Naciones Unidas para los Derechos Humanos. Bogotá, 41.

De Chazal Palomo, J. y Saucedo Justiniano, J, (1998). Declaraciones fundamentales y Derechos constitucionales, Santa Cruz, UPSA. 
Deuteronomio: 25.3

Diez - Picazo, Luis María, (2000). "Aproximación a la idea de los derechos fundamentales", Lima, Revista Peruana de Derecho Constitucional, pp. 221 y ss.

Echeburúa, E. y Paz de Corral (2005). ¿Cómo evaluar las lesiones psíquicas y las secuelas emocionales en las víctimas de delitos violentos? Psicopatología Clínica, Legal y Forense, Vol. 5., 2005, pp 57-73. Universidad del País Vasco. España.

Echeburúa, E., Corral, P. y Amor, P.J. (1998). Perfiles diferenciales del trastorno de estrés postraumático en distintos tipos de víctimas. Análisis y Modificación de Conducta, 24, 527-555.

Esbec, E. (2000). Evaluación psicológica de la víctima. En E. Esbec y G. Gómez-Jarabo. Psicología forense y tratamiento jurídico-legal de la discapacidad. Madrid. Edisofer.

Estela, J. (2011). El proceso de amparo como mecanismo de tutela de los derechos procesales. Tesis para optar el grado académico de Magíster en Derecho Constitucional y Derechos Humanos. Unidad de Postgrado. Universidad Nacional Mayor de San Marcos. Lima.

Ferrajoli, L. (1997). Derechos y Garantías, Madrid. Ed. Trotta S.A.

Ferreira, G. (1989). La mujer maltratada. Buenos Aires: Sudamérica.

Foucault, M. (1985), Vigilar y Castigar. Nacimiento de la prisión. México: Siglo XXI Editores, 45.

Fuentes, P. (2016). Factores intrafamiliares y jurídicos asociados a la violencia familiar. Distrito de Tacna. 2015. Tesis para optar el grado de Maestro en derecho civil y comercial. Escuela de Posgrado. Universidad Nacional Jorge Basadre Grohmann. Tacna.

García-Montoya, L. (2011). Criminología y violencia familiar: una aproximación a la violencia en el hogar a partir del estudio de las características del maltratador. Tesis doctoral. Centro de Investigación en Criminología. Universidad Castilla-La Mancha. España.

Gonzales, G. (2016). Incumplimiento de las medidas de protección dictadas al amparo del artículo 10 de la ley de protección frente a la violencia familiar, según los procesos de ejecución de sentencia en violencia familiar del segundo y cuarto juzgado de familia de la corte superior de justicia de Arequipa, de enero del 2010 a diciembre del 2011. Tesis de maestría de derecho civil. Universidad Católica Santa María, Arequipa.

Hirigoyen, M.F. (1999). El acoso moral. Barcelona: Edit. Paidós

Jacobson, N. y Gottman, J. (2001). Hombres que agreden a sus mujeres: cómo poner fin a las relaciones abusivas. México: Edit. Paidós

Jaramillo Vélez, R. (1996), Historia de los Derechos Humanos en La responsabilidad en Derechos Humanos. Universidad Nacional, 156.

Labrador, F., Paz, P., De Luis, P., Fernández-Velasco, R. (2004). Mujeres víctimas de la violencia doméstica. Madrid: Programas de Actuación

Laguna, G. (2015). Los procesos ante los juzgados de violencia sobre la mujer. Memoria para optar al grado de doctor. Facultad de derecho. Universidad complutense de Madrid. España.

Lorente, M. (2005). Violencia contra las mujeres y trato indigno. Entre la invisibilidad y la negación. En: Mañas, C. (2008). Violencia Estructural y Directa: Mujeres y Visibilidad. FEMINISMO/S, 6. Alicante. Editorial: Centro de Estudios sobre la Mujer de la Universidad de Alicante (CEM). España.

Lin Ching, R. (2003). Propuesta de valoración del daño psicológico en materia de violencia doméstica. Costa Rica.

Lujan, M. (2013). Violencia contra las mujeres y alguien más. Tesis doctoral Universitat de València. (Estudi General). Facultad de Derecho. Departamento de Filosofía del Derecho, Moral y Política. España.

Magaña de la Mora, M. (2017). El delito de violencia familiar: un estudio comparativo de la situación en España y el Estado de Michoacán (México). Memoria para optar el grado de Doctor en Derecho. Universidad Complutense de Madrid. España.

Martínez-Pujalte, A. (1992). "Los Derechos Humanos como Derechos Inalienables", en Jesús Ballesteros, Derechos Humanos: Concepto, Fundamentos, Sujetos, Madrid, Tecnos, 92. 